\title{
RelocaTE2: a high resolution transposable element insertion site mapping tool for population resequencing
}

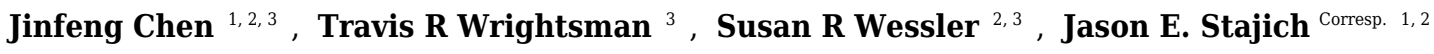 \\ 1 Department of Plant Pathology \& Microbiology, University of California, Riverside, Riverside, CA, United State \\ 2 Institute for Integrative Genome Biology, University of California, Riverside, Riverside, CA, United States \\ 3 Department of Botany and Plant Sciences, University of California, Riverside, Riverside, CA, United States \\ Corresponding Author: Jason E. Stajich \\ Email address: jason.stajich@ucr.edu
}

Background Transposable element (TE) polymorphisms are important components of population genetic variation. The functional impacts of TEs in gene regulation and generating genetic diversity have been observed in multiple species, but the frequency and magnitude of TE variation is under appreciated. Inexpensive and deep sequencing technology has made it affordable to apply population genetic methods to whole genomes with methods that identify single nucleotide and insertion/deletion polymorphisms. However, identifying TE polymorphisms, particularly transposition events or nonreference insertion sites can be challenging due to the repetitive nature of these sequences, which hamper both the sensitivity and specificity of analysis tools.

Methods We have developed the tool RelocaTE2 ( http://github.com/stajichlab/RelocaTE2 ) for identification of TE insertion sites at high sensitivity and specificity. RelocaTE2 searches for known TE sequences in whole genome sequencing reads from second generation sequencing platforms such as Illumina. These sequence reads are used as seeds to pinpoint chromosome locations where TEs have transposed. RelocaTE2 detects target site duplication (TSD) of TE insertions allowing it to report TE polymorphism loci with single base pair precision.

Results and Discussion The performance of RelocaTE2 is evaluated using both simulated and real sequence data. RelocaTE2 demonstrate high level of sensitivity and specificity, particularly when the sequence coverage is not shallow. In comparison to other tools tested, RelocaTE2 achieves the best balance between sensitivity and specificity. In particular, RelocaTE2 performs best in prediction of TSDs for TE insertions. Even in highly repetitive regions, such as those tested on rice chromosome 4, RelocaTE2 is able to report up to $95 \%$ of simulated TE insertions with less than $0.1 \%$ false positive rate using 10 -fold genome coverage resequencing data. RelocaTE2 provides a robust solution to identify TE insertion sites and can be incorporated into analysis workflows in support of describing the complete genotype from light coverage genome sequencing. 
1 RelocaTE2: a high resolution transposable element insertion site mapping tool for 2 population resequencing

3 Jinfeng Chen ${ }^{1,2,3}$, Travis R. Wrightsman ${ }^{2}$, Susan R. Wessler ${ }^{2,3}$ and Jason E. Stajich ${ }^{1,3, *}$

$4{ }^{1}$ Department of Plant Pathology and Microbiology, University of California-Riverside, 5 Riverside CA 92521, USA.

62 Department of Botany and Plant Sciences, University of California-Riverside, Riverside CA 792521 , USA.

$8{ }^{3}$ Institute for Integrative Genome Biology, University of California-Riverside, Riverside, CA 9 92521, USA.

10 *To whom correspondence should be addressed. E-mail: jason.stajich@ucr.edu 


\section{Abstract}

\section{Background}

13 Transposable element (TE) polymorphisms are important components of population genetic

14 variation. The functional impacts of TEs in gene regulation and generating genetic diversity have

15 been observed in multiple species, but the frequency and magnitude of TE variation is under

16 appreciated. Inexpensive and deep sequencing technology has made it affordable to apply

17 population genetic methods to whole genomes with methods that identify single nucleotide and

18 insertion/deletion polymorphisms. However, identifying TE polymorphisms, particularly

19 transposition events or non-reference insertion sites can be challenging due to the repetitive

20 nature of these sequences, which hamper both the sensitivity and specificity of analysis tools.

\section{Methods}

22 We have developed the tool RelocaTE2 (http://github.com/stajichlab/RelocaTE2) for

23 identification of TE insertion sites at high sensitivity and specificity. RelocaTE2 searches for

24 known TE sequences in whole genome sequencing reads from second generation sequencing

25 platforms such as Illumina. These sequence reads are used as seeds to pinpoint chromosome

26 locations where TEs have transposed. RelocaTE2 detects target site duplication (TSD) of TE

27 insertions allowing it to report TE polymorphism loci with single base pair precision.

\section{Results and Discussion}

29 The performance of RelocaTE2 is evaluated using both simulated and real sequence data.

30 RelocaTE2 demonstrate high level of sensitivity and specificity, particularly when the sequence

31 coverage is not shallow. In comparison to other tools tested, RelocaTE2 achieves the best

32 balance between sensitivity and specificity. In particular, RelocaTE2 performs best in prediction

33 of TSDs for TE insertions. Even in highly repetitive regions, such as those tested on rice

34 chromosome 4, RelocaTE2 is able to report up to $95 \%$ of simulated TE insertions with less than

$350.1 \%$ false positive rate using 10-fold genome coverage resequencing data. RelocaTE2 provides

36 a robust solution to identify TE insertion sites and can be incorporated into analysis workflows in

37 support of describing the complete genotype from light coverage genome sequencing. 


\section{Introduction}

39 Transposable elements (TE), mobile DNA of the genome, are drivers of genomic innovation 40 (Bennetzen \& Wang 2014; Cordaux \& Batzer 2009). They can act as mutagens to disrupt gene 41 functions or induce novel gene functions by providing enhancers or promoters that alter host 42 gene expression (Feschotte 2008; Lisch 2013). In plants, TEs have been shown to contribute to 43 several key trait innovations in crop domestication (Lisch 2013). Systematic analysis of TE 44 insertions and gene expression also suggests widespread roles of TEs in altering gene regulation (Kunarso et al. 2010; Lynch et al. 2011; Sundaram et al. 2014). It was found that 600-2000 genetic variants between individuals in the human population and 200-300 variants between Arabidopsis accessions could be attributed to TE polymorphisms (Quadrana et al. 2016; Stewart et al. 2011). Although the magnitude of these polymorphisms is small compared to SNPs or other insertion/deletions, some TE polymorphisms are proximal to protein coding genes and can 50 have large impacts on gene function or gene regulation (Cowley \& Oakey 2013; Quadrana et al. 51 2016; Stewart et al. 2011).

52 Two categories of bioinformatics tools have been developed to identify TE polymorphisms from 53 population resequencing data. One type employs a strategy similar to that used to discover 54 structural variations. These tools identify discordant pairs of sequence reads based on the 55 56 57 chromosomal position of read alignments to indicate genomic inversions, insertions, deletions or other complex rearrangements (Campbell et al. 2008; Korbel et al. 2007). Software for TE mapping scrutinize genomic loci with discordant read pairs to see if known TE sequences are can be implicated near the rearrangement site. These tools, such as Retroseq (Keane et al. 2013) and TEMP (Zhuang et al. 2014), are generally highly sensitive and can locate TE insertions and absences to a 10-50 bp resolution. A second category of tools operates by first identifying by similarity any sequence reads containing partial or complete known TE sequences. Any sequence containing a TE is a "junction-read" which contains partial TE and partial unique host genomic sequence. These tools eliminate the TE sequence from these junction-reads and search the remaining $5^{\prime}$ or $3^{\prime}$ flanking sequence against the host organism genome sequence to identify the element's location. These junctions-based tools, including RelocaTE (Robb et al. 2013) and ITIS (Jiang et al. 2015), are able to detect the exact location and TSD characteristic of TE insertion sites. This second category of tools is ideal for identifying new insertions from population resequencing data because it can accurately detect an insertion location along with the TSD. 
69 However, most of these tools are designed to search a single transposable element representative 70 sequence at a time, which sacrifices speed for increased sensitivity and specificity. The extended 71 runtime limits the feasibility of applying these tools when searching thousands of TEs in

72 hundreds or thousands of individuals.

73 RelocaTE2 is an improved version of RelocaTE where we have implemented a junction-based 74 approach that can search multiple template TEs in the same pass through short read sequencing 75 data, streamlining the computational approach. Using simulated datasets, we show that 76 RelocaTE2 is highly sensitive even in low coverage resequencing data or on chromosomes with 77 a high repetitive sequence content with a specificity of greater than $99 \%$. In comparing 78 performance of related tools, RelocaTE2 was the most sensitive and specific tool in our tests 79 profiling human and rice population genomics data. The tool is presented as a useful resource for 80 analyzing population dynamics of TEs in genomic resequencing data.

\section{Materials \& Methods}

82 RelocaTE2 Workflow

83 RelocaTE2 is based on the previous algorithm implemented in RelocaTE (Robb et al. 2013), 84 which uses junction reads to find insertion sites of TEs. In RelocaTE2, we re-implement the 85 search strategy to enable identification of multiple TEs in a single search, greatly increasing the speed and enabling searches for hundreds or thousands of candidate TE families in a genome

87 (Fig. 1). We also implement new features in the algorithm to automatically identify TSDs and remove false junction reads (Fig. 1).

89 Briefly, the workflow initiates by matching a library of known repeat elements against short 90 sequence reads generated by next generation sequencing, typically Illumina paired-end reads, 91 using BLAT with the sensitive setting "-minScore=10 -tileSize=7" (Kent 2002; Robb 92 et al. 2013). Every read with similarity to repeat elements is denoted as an informative read and 93 will contain a partial or complete copy of a TE. Informative reads that contain partial matches at 94 the boundaries of the repeat elements are trimmed to remove the TE region so that only the 95 regions flanking the element remain in either one or both of the paired-end reads (denoted as 96 junction reads). Untrimmed versions of each junction read and its pair (denoted as full reads) are 97 used as controls to filter false positive junction reads. 
98 Sequence reads comprised entirely of repeat elements are ignored, but their read pair is kept

99 (denoted as supporting reads). These junction, full, and supporting reads, are all aligned to the 100 reference genome using BWA (v0.6.2) with the default setting "-1 32 - k 2" (Li \& Durbin

101 2009). Mapped reads are sorted by chromosome order and windows of 2,000 bp are evaluated to

102 define insertion clusters. In each insertion cluster, additional subclusters are further refined based

103 on the mapping position of junction reads to address the possible scenario of multiple insertion

104 sites within a window. TSD position and sequence are identified if the subcluster is supported by

105 junction reads from both upstream and downstream of the TE insertion site.

106 Next, a series of cleaning steps are used to filter low quality candidate insertion sites: i.) remove

107 insertion sites that are only supported by low quality junction reads (map quality < 29); ii.)

108 remove insertion site only supported by less than 3 junction reads total on the left or right flank

109 when there are additional insertion sites which pass these filters in the same window. iii.) remove

110 insertion sites only supported by junction reads and located within $10 \mathrm{bp}$ range of an annotated

111 TE in the reference genome. RelocaTE2 output reports the number of junction reads and

112 supporting reads from both upstream and downstream of candidate TE insertion sites. Only

113 confident insertions, defined as having at least one supporting junction read flanking the

114 upstream or downstream of insertion sites and at least one junction read or one supporting read

115 supporting the other end of TE insertion, are provided in the default output:

116 "ALL.all_nonref_insert.gff". Additional information about all candidate sites are provided in

117 alternative output file: "ALL.all_nonref_insert.raw.gff".

\section{Simulated data for evaluation of TE insertion tools}

119 Simulated datasets were created by randomly inserting TEs into sequence records of

120 chromosomes 3 (OsChr3) and 4 (OsChr4) of rice (Oryza sativa japonica). OsChr3 is primarily

121 made up of euchromatic regions, whereas OsChr4 has the sequence complexity consistent with

122 heterochromatic regions and is a typical feature of many plant genomes (Zhao et al. 2002).

123 Fourteen TEs families found in rice genomes comprised of 7 DNA Transposons: $m$ Ping, $n$ Dart,

124 Gaijin, spmlike, Truncator, mGing, nDarz and 7 RNA Retrotransposons: Bajie, Dasheng,

125 Retro1, RIRE2, RIRE3, Copia2, karma, were used. The insertion simulations were performed by

126 choosing 200 random insertion sites on each chromosome in three independent replicates. Each

127 simulated insertion site was generated by selecting one random chromosome position, one

128 random TE, and inserting the element along with the expected TSDs. After generating 200 
129 random insertions, a new genome sequence was generated along with a GFF3 file containing the

130 recorded insertion locations to support the performance evaluation of the dataset. Paired-end

131 reads of all simulated chromosomes were simulated by pIRS (pirs simulate -1100 -x coverage -

$132 \mathrm{~m} 500-\mathrm{v} 100)$ (Hu et al. 2012). For each dataset, simulate sequence reads at sequence depths of

$1331,2,3,4,5,6,7,8,9,10,15,20,40$-fold coverage were generated.

\section{Real sequence data for evaluation of TE insertion tools}

135 Three sets of data, an individual human genome, HuRef, an individual rice genome, IR64, and

136 population resequencing data of 50 rice and wild rice genomes (Levy et al. 2007; Schatz et al.

137 2014; Xu et al. 2012), were used to evaluate the performance of RelocaTE2 and TEMP. High

138 quality genome assemblies of HuRef and IR64 were used to evaluate the accuracy of TE

139 genotyping tools by comparing the assembled sequences to the reference genome. The HuRef

140 (also known as Venter genome) has been extensively studied for TE insertions (Xing et al.

141 2009). Previous work identified 574 Alu elements that have been experimentally verified and can

142 be treated as a gold standard data set for evaluation (Hormozdiari et al. 2010; Xing et al. 2009).

143 Paired-end sequence reads of 10-fold depth were simulated from HuRef as test dataset by pIRS

144 (pirs simulate -l 100 -x coverage -m 500 -v 100) (Hu et al. 2012).

145 RelocaTE2 and TEMP were tested and their results compared to the Human Genome Reference

146 Consortium genome (GRCh36 or hg18). A second dataset, the finished reference genome

147 assembly of rice strain IR64, was explored utilizing available Illumina sequencing reads (Schatz

148 et al. 2014). RelocaTE2 and TEMP were tested on 20-fold genome coverage of 100 bp paired-

149 end Illumina short reads (SRA accession: SRR546439) aligned to the rice reference genome

150 (MSU7). A third dataset of resequencing data from 50 strains of rice and wild rice population

151 with an average sequencing depth of 17-fold (Xu et al. 2012). RelocaTE2 and TEMP were tested

152 on the sequencing libraries from each of these 50 strains to assess their consistency across

153 datasets with varying sequence depth and genetic diversity. RelocaTE and ITIS were not

154 included in the biological data testing because of the prohibitively long run times on these large

155 datasets and their poor performance on simulated datasets.

156 Detection of TE insertions using RelocaTE2, RelocaTE, TEMP and ITIS

157 RelocaTE2, RelocaTE, TEMP and ITIS were run with default parameter settings on simulated

158 data. The results were filtered to evaluate the best performing parameters for each tool.

159 RelocaTE2 was tested with parameters "--len_cut_match 10 --len_cut_trim 10 
160 --mismatch 2 --aligner blat", which uses BLAT as the search engine (--aligner

$161 \mathrm{blat}$ ), allows for 2 mismatches (- - mismatch 2) in matched sequence between reads and

162 repeat elements (--len_cut_match 10), and only keeps sequence fragments that have at

163 least $10 \mathrm{bp}$ after trimming repeat elements from reads (--len_cut_trim 10). RelocaTE

164 was tested using parameters "--len_cutoff 10 --mismatch 0", which uses BLAT as

165 search engine by default and allowed $0 \mathrm{bp}$ mismatch (- -mismatch 0$)$ for matched sequence

166 between reads and repeat elements (--len_cutoff 10$)$. It should be noted that the mismatch

167 setting in RelocaTE is the ratio of base pairs in the alignment between reads and repeat elements

168 that can be mismatched, not an integer number of allowed mismatches, as used in RelocaTE2.

169 Singleton calls from RelocaTE's results, which are sites supported by only one read, were

170 removed. TEMP was tested with parameters "-m 3", which allow for three mismatches

171 between reads and repeat elements. Singleton calls from TEMP's results were removed when

172 testing on simulated data to achieve a balance between sensitivity and specificity. ITIS was

173 tested with default parameters, which filtered TE calls with at least one read supporting from

174 both ends of TE insertions. For analysis of the HuRef genome, the IR64 genome and the 50 rice

175 and wild rice strains, RelocaTE2 and TEMP were run with default parameter settings as

176 described above. The TEMP results were filtered to keep only TE calls with supporting and/or

177 junction reads from both ends of TE insertions to achieve a comparable balance between

178 sensitivity and specificity.

179 Results and Discussions

\section{Performance of RelocaTE2, RelocaTE, TEMP and ITIS on simulated data}

181 RelocaTE2 was first compared to RelocaTE, TEMP and ITIS using the simulated datasets. Each

182 dataset of simulated rice chromosomes, OsChr3 and OsChr4, was virtually sheared to simulated

183 paired-end short reads at a coverage ranging from 1 to 40 -fold. At high sequencing coverage

184 ( $\geq 10$-fold), RelocaTE2, TEMP and ITIS were able to identify $>99 \%$ of simulated insertions on

185 OsChr3, whereas the performance of RelocaTE was much lower (85\%) (Fig. 2A). At lower

186 sequencing coverage, e.g. 3-fold, only RelocaTE2 and TEMP were able to achieve $\geq 95 \%$

187 sensitivity on OsChr3 (Fig. 2A). Furthermore, TEMP was able to identify 83\% and 93\% of

188 simulated insertions on $\mathrm{OsChr} 3$ at very low sequence coverage of 1-fold and 2-fold, respectively 189 (Fig. 
190 2A). RelocaTE2 had a sensitivity of $53 \%$ and $83 \%$ on OsChr3 for the 1 -fold and 2 -fold coverage

191 due to the removal of TE insertions supported by only one read (singleton) or supported by reads

192 from only one end of TE insertions (insufficient insertions), which can result in many false

193 positives (Fig. 2A).

194 RelocaTE2, RelocaTE and TEMP showed $>99 \%$ specificity on OsChr3 at multiple levels of 195 sequence coverage (Fig. 2B). In contrast, the specificity of ITIS was much lower (<90\%), even

196 when run on the high sequence coverage dataset on OsChr3 (Fig. 2B). In comparing recall rates

197 of TSDs, RelocaTE2 and ITIS had similar performance and achieved the highest recall rate of

$19898 \%$ and 91\% respectively, on OsChr3 at $\sim 10$-fold coverage (Fig. 2C). The recall rate of TSDs

199 for both TEMP and RelocaTE depended on sequence depth and achieved only $37 \%$ and $60 \%$,

200 respectively, at 10-fold coverage (Fig. 2C). All the tools performed worse on OsChr4 as

201 compared to OsChr3 (Fig. 2D-F). RelocaTE2 demonstrated a lower average sensitivity (92\%) on

202 OsChr4 when compared OsChr3 (96\%) (Fig. 2A, D). Similarly, TEMP had a slightly lower

203 sensitivity (95\%) on OsChr4 than on OsChr3 (97\%) (Fig. 2A, D). However, RelocaTE2 and

204 RelocaTE demonstrated high level of the specificity ( $>99 \%$ ) while TEMP performed at a slightly

205 lower specificity (98\%) on OsChr4 compared to $>99 \%$ on OsChr3 (Fig. 2B, E). In comparing

206 TSD accuracy on OsChr4, on average 81\% of RelocaTE2 calls correctly identified the TSD,

207 whereas only $31 \%$ of TEMP calls were correct (Fig. 2C, F).

208 Evaluation of RelocaTE2 and TEMP on biological datasets

209 We evaluated TE identifying tools in the HuRef genome and benchmark the sensitivity and 210 specificity of these tools using 574 experimental verified Alu insertions in HuRef genome and

211 genomic comparison between HuRef genome and GRCh36. RelocaTE2 and TEMP reported 212 similar results and identified 83\% (479/574) and 76\% (438/574) of standard insertion sites (Fig.

213 3A). Comparing the HuRef genome with GRCh36 suggested that $89 \%$ and $95 \%$ of insertions 214 identified by RelocaTE2 and TEMP, respectively, were real insertions (Fig. 3A). In addition,

215 RelocaTE2 predicted TE insertion sites with higher precision ( $9 \pm 6 \mathrm{bp}$ ) compared to TEMP (366 $216 \pm 170 \mathrm{bp})$.

217 RelocaTE2 and TEMP were used to analyze data from the rice strain IR64 and the results were 218 evaluated by comparing the genome assembly of IR64 with MSU7. RelocaTE2 identified 648 219 insertion sites while the genome comparison revealed that $93 \%$ of insertions were true positives 220 (Fig. 3A). TEMP identified 362 insertions, of which 50\% (183/362) overlapped with RelocaTE2 
221 (Fig. 3A). The specificity of TEMP was estimated to be $86 \%$, slightly lower than RelocaTE2

222 (93\%) (Fig. 3A). However, TEMP was found to be less sensitive than RelocaTE2 in the rice 223 genome, only calling 362 sites as compared to 648 by RelocaTE2 (38\% vs. 90\%, Fig. 3A).

224 Moreover, RelocaTE2 predicted TE insertion junctions of $3 \pm 1 \mathrm{bp}$, which was much smaller 225 than TEMP $(393 \pm 199 \mathrm{bp})$.

226 RelocaTE2 and TEMP were used to identify TE polymorphisms in 50 resequenced rice and wild 227 rice strains, which contain substantial sequence diversity and population structure (Xu et al. 228 2012). The results from these two tools were well correlated $\left(R^{2}=0.96, P\right.$ value $\left.=2.2 \mathrm{e}-16\right)$ and 229 predicted more TE insertions in the diverged population of wild rice, O. nivara and O. rufipogon, 230 and even in the indica population than japonica rice which close to the reference genome (Fig. 231 3B). On average $72 \%$ of the sites predicted in these 50 rice and wild rice strain by RelocaTE2 232 and TEMP overlapped. Many insertion sites from TEMP were predicted with only supporting 233 read flanking one end of an insertion, which produced large variations in predicted junctions of

234 TE insertion sites $(118 \pm 151 \mathrm{bp})$. In contrast, RelocaTE2 reported most of TE insertions

235 supported by junction reads or supporting reads on both ends, which resulted in accurate 236 insertion junction predictions $(3 \pm 2 \mathrm{bp})$.

\section{Runtime performance}

238 We implemented the searching process for TE insertion to run on multiple processors in Python.

239 The process is relatively memory efficient. When searching TEs in the rice genome for example, 240 one process generally uses less than $1 \mathrm{~Gb}$ memory. The running time of RelocaTE2 depends on 241 number of processors used. Searching 3000 templates of transposable elements with 20-fold 242 genome coverage sequencing data of the rice genome takes 3-4 hours for RelocaTE2 using 32 243 CPUs including the alignment steps. TEMP identifies transposable element insertions from a 244 BAM file. It takes $\sim 1$ hours for TEMP for the same project using single process. RelocaTE 245 (version 1) and ITIS take at least days for the same rice datasets and can be prohibitively difficult 246 to run on large datasets with multiple templates due to the serial searching approach of their 247 implementation.

\section{Conclusions}

250 We present RelocaTE2 as a new tool for mapping TE insertions to base-pair resolution from 251 resequencing data. RelocaTE2 identifies multiple TE families in a single search with high 
252 sensitivity and specificity. The evaluation of these tools on simulated and biological datasets

253 support the use of RelocaTE2 for analysis of genomes of plants and animals and indicate it can

254 generate very high quality genotyping of TE insertions from resequencing datasets of modest

255 sequencing depths. TEMP is fast and achieves well balance between sensitivity and specificity in

256 identifying TE polymorphisms, particularly when the sequence depth is shallow. Although the

257 precision of TSD prediction of TEMP is not very sensitive compared to RelocaTE2 and other

258 tools. We recommend using TEMP in scenarios when accuracy of TSD is not very critical. The

259 high resolution mapping of TE insertions sites will enable detailed analysis of the interaction of

260 TEs and genes and as structural variations that vary in populations.

261

262 Data Availability

263 The source code in Python, manual, and sample data of RelocaTE2 are available for download at

264 https://github.com/stajichlab/RelocaTE2.

265

266

267

268

269

270

271

272

273

274

275

276

277

278

279

280

281

282

283

284

285

286

287

288

289

\section{References}

Bennetzen JL, and Wang H. 2014. The contributions of transposable elements to the structure, function, and evolution of plant genomes. Annu Rev Plant Biol 65:505-530. 10.1146/annurev-arplant-050213-035811

Campbell PJ, Stephens PJ, Pleasance ED, O'Meara S, Li H, Santarius T, Stebbings LA, Leroy C, Edkins S, Hardy C, Teague JW, Menzies A, Goodhead I, Turner DJ, Clee CM, Quail MA, Cox A, Brown C, Durbin R, Hurles ME, Edwards PA, Bignell GR, Stratton MR, and Futreal PA. 2008. Identification of somatically acquired rearrangements in cancer using genome-wide massively parallel paired-end sequencing. Nat Genet 40:722729. 10.1038/ng.128

Cordaux R, and Batzer MA. 2009. The impact of retrotransposons on human genome evolution. Nat Rev Genet 10:691-703. 10.1038/nrg2640

Cowley M, and Oakey RJ. 2013. Transposable elements re-wire and fine-tune the transcriptome. PLoS Genet 9:e1003234. 10.1371/journal.pgen.1003234

Feschotte C. 2008. Transposable elements and the evolution of regulatory networks. Nat Rev Genet 9:397-405. 10.1038/nrg2337

Hormozdiari F, Hajirasouliha I, Dao P, Hach F, Yorukoglu D, Alkan C, Eichler EE, and Sahinalp SC. 2010. Next-generation VariationHunter: combinatorial algorithms for transposon insertion discovery. Bioinformatics 26:i350-357. 10.1093/bioinformatics/btq216

Hu X, Yuan J, Shi Y, Lu J, Liu B, Li Z, Chen Y, Mu D, Zhang H, Li N, Yue Z, Bai F, Li H, and Fan W. 2012. pIRS: Profile-based Illumina pair-end reads simulator. Bioinformatics 28:1533-1535. 10.1093/bioinformatics/bts187 
290 Jiang C, Chen C, Huang Z, Liu R, and Verdier J. 2015. ITIS, a bioinformatics tool for accurate

291

292

293

294

295

296

297

298

299

300

301

302

303

304

305

306

307

308

309

310

311

312

313

314

315

316

317

318

319

320

321

322

323

324

325

326

327

328

329

330

331

332

333

334

335 identification of transposon insertion sites using next-generation sequencing data. BMC Bioinformatics 16:72. 10.1186/s12859-015-0507-2

Keane TM, Wong K, and Adams DJ. 2013. RetroSeq: transposable element discovery from next-generation sequencing data. Bioinformatics 29:389-390.

10.1093 /bioinformatics/bts697

Kent WJ. 2002. BLAT--the BLAST-like alignment tool. Genome Res 12:656-664. 10.1101/gr.229202. Article published online before March 2002

Korbel JO, Urban AE, Affourtit JP, Godwin B, Grubert F, Simons JF, Kim PM, Palejev D, Carriero NJ, Du L, Taillon BE, Chen Z, Tanzer A, Saunders AC, Chi J, Yang F, Carter NP, Hurles ME, Weissman SM, Harkins TT, Gerstein MB, Egholm M, and Snyder M. 2007. Paired-end mapping reveals extensive structural variation in the human genome. Science 318:420-426. 10.1126/science.1149504

Kunarso G, Chia NY, Jeyakani J, Hwang C, Lu X, Chan YS, Ng HH, and Bourque G. 2010. Transposable elements have rewired the core regulatory network of human embryonic stem cells. Nat Genet 42:631-634. 10.1038/ng.600

Levy S, Sutton G, Ng PC, Feuk L, Halpern AL, Walenz BP, Axelrod N, Huang J, Kirkness EF, Denisov G, Lin Y, MacDonald JR, Pang AW, Shago M, Stockwell TB, Tsiamouri A, Bafna V, Bansal V, Kravitz SA, Busam DA, Beeson KY, McIntosh TC, Remington KA, Abril JF, Gill J, Borman J, Rogers YH, Frazier ME, Scherer SW, Strausberg RL, and Venter JC. 2007. The diploid genome sequence of an individual human. PLoS Biol 5:e254. 10.1371/journal.pbio.0050254

Li H, and Durbin R. 2009. Fast and accurate short read alignment with Burrows-Wheeler transform. Bioinformatics 25:1754-1760. 10.1093/bioinformatics/btp324

Lisch D. 2013. How important are transposons for plant evolution? Nat Rev Genet 14:49-61. $10.1038 / \mathrm{nrg} 3374$

Lynch VJ, Leclerc RD, May G, and Wagner GP. 2011. Transposon-mediated rewiring of gene regulatory networks contributed to the evolution of pregnancy in mammals. Nat Genet 43:1154-1159. 10.1038/ng.917

Quadrana L, Bortolini Silveira A, Mayhew GF, LeBlanc C, Martienssen RA, Jeddeloh JA, and Colot V. 2016. The Arabidopsis thaliana mobilome and its impact at the species level. Elife 5. 10.7554/eLife.15716

Robb SM, Lu L, Valencia E, Burnette JM, 3rd, Okumoto Y, Wessler SR, and Stajich JE. 2013. The use of RelocaTE and unassembled short reads to produce high-resolution snapshots of transposable element generated diversity in rice. G3 (Bethesda) 3:949957. 10.1534/g3.112.005348

Schatz MC, Maron LG, Stein JC, Hernandez Wences A, Gurtowski J, Biggers E, Lee H, Kramer M, Antoniou E, Ghiban E, Wright MH, Chia JM, Ware D, McCouch SR, and McCombie WR. 2014. Whole genome de novo assemblies of three divergent strains of rice, Oryza sativa, document novel gene space of aus and indica. Genome Biol 15:506. 10.1186/PREACCEPT-2784872521277375

Stewart C, Kural D, Stromberg MP, Walker JA, Konkel MK, Stutz AM, Urban AE, Grubert F, Lam HY, Lee WP, Busby M, Indap AR, Garrison E, Huff C, Xing J, Snyder MP, Jorde LB, Batzer MA, Korbel JO, Marth GT, and Genomes P. 2011. A comprehensive map of mobile element insertion polymorphisms in humans. PLoS Genet 7:e1002236. 10.1371/journal.pgen.1002236 
336

337

338

339

340

341

342

343

344

345

346

347

348

349

350

351

352

353

354

355

Sundaram V, Cheng Y, Ma Z, Li D, Xing X, Edge P, Snyder MP, and Wang T. 2014. Widespread contribution of transposable elements to the innovation of gene regulatory networks. Genome Res 24:1963-1976. 10.1101/gr.168872.113

Xing J, Zhang Y, Han K, Salem AH, Sen SK, Huff CD, Zhou Q, Kirkness EF, Levy S, Batzer MA, and Jorde LB. 2009. Mobile elements create structural variation: analysis of a complete human genome. Genome Res 19:1516-1526. 10.1101/gr.091827.109

Xu X, Liu X, Ge S, Jensen JD, Hu F, Li X, Dong Y, Gutenkunst RN, Fang L, Huang L, Li J, He W, Zhang G, Zheng X, Zhang F, Li Y, Yu C, Kristiansen K, Zhang X, Wang J, Wright M, McCouch S, Nielsen R, Wang J, and Wang W. 2012. Resequencing 50 accessions of cultivated and wild rice yields markers for identifying agronomically important genes. Nat Biotechnol 30:105-111. 10.1038/nbt.2050

Zhao Q, Zhang Y, Cheng Z, Chen M, Wang S, Feng Q, Huang Y, Li Y, Tang Y, Zhou B, Chen Z, Yu S, Zhu J, Hu X, Mu J, Ying K, Hao P, Zhang L, Lu Y, Zhang LS, Liu Y, Yu Z, Fan D, Weng Q, Chen L, Lu T, Liu X, Jia P, Sun T, Wu Y, Zhang Y, Lu Y, Li C, Wang R, Lei H, Li T, Hu $\mathrm{H}$, Wu M, Zhang R, Guan J, Zhu J, Fu G, Gu M, Hong G, Xue Y, Wing R, Jiang J, and Han B. 2002. A fine physical map of the rice chromosome 4. Genome Res 12:817-823. $10.1101 /$ gr.48902

Zhuang J, Wang J, Theurkauf W, and Weng Z. 2014. TEMP: a computational method for analyzing transposable element polymorphism in populations. Nucleic Acids Res 42:6826-6838. 10.1093/nar/gku323 


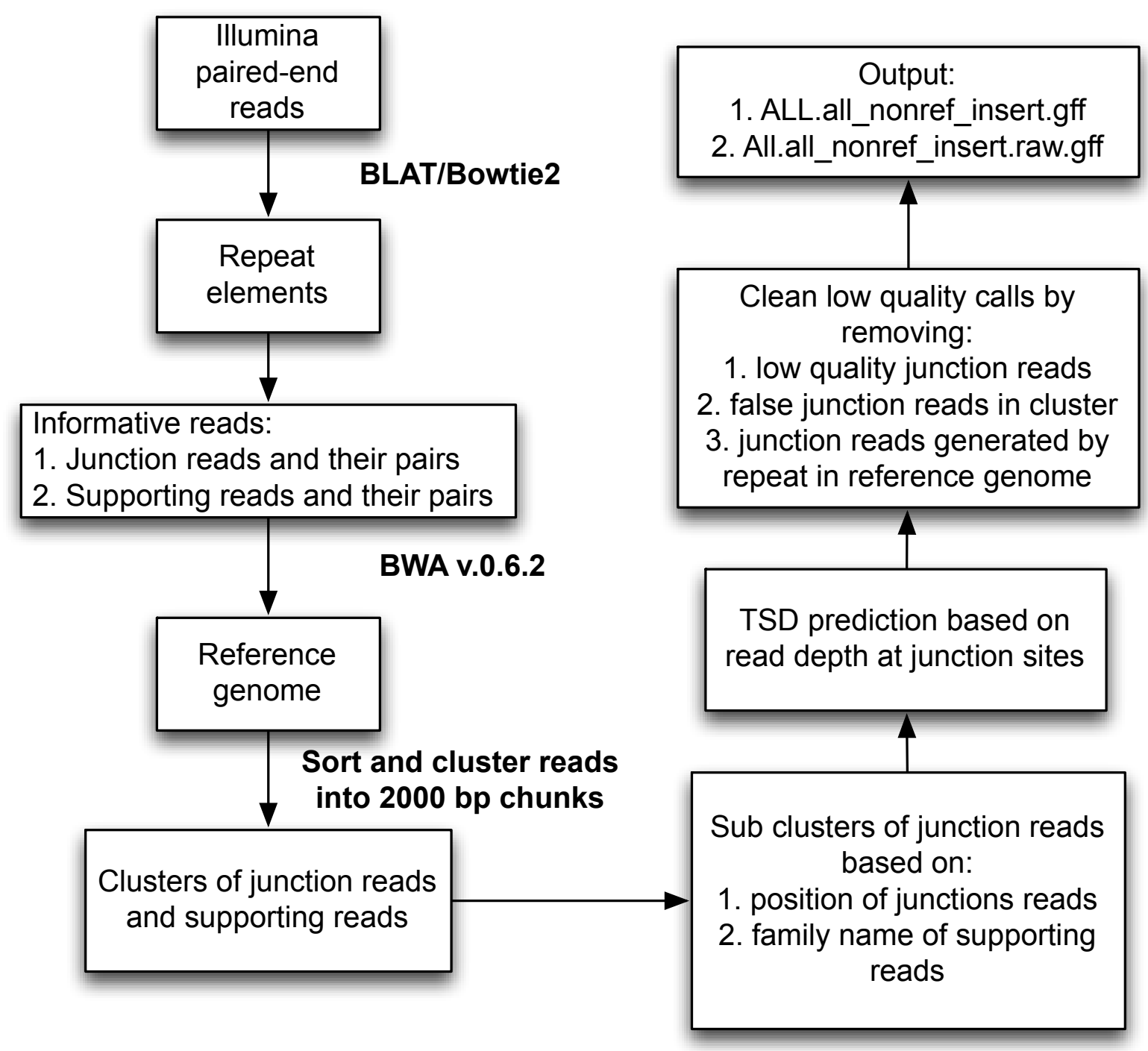

358 Figure 1. Workflow for identification of transposable element insertions in population resequencing data using Illumina paired-end reads. 

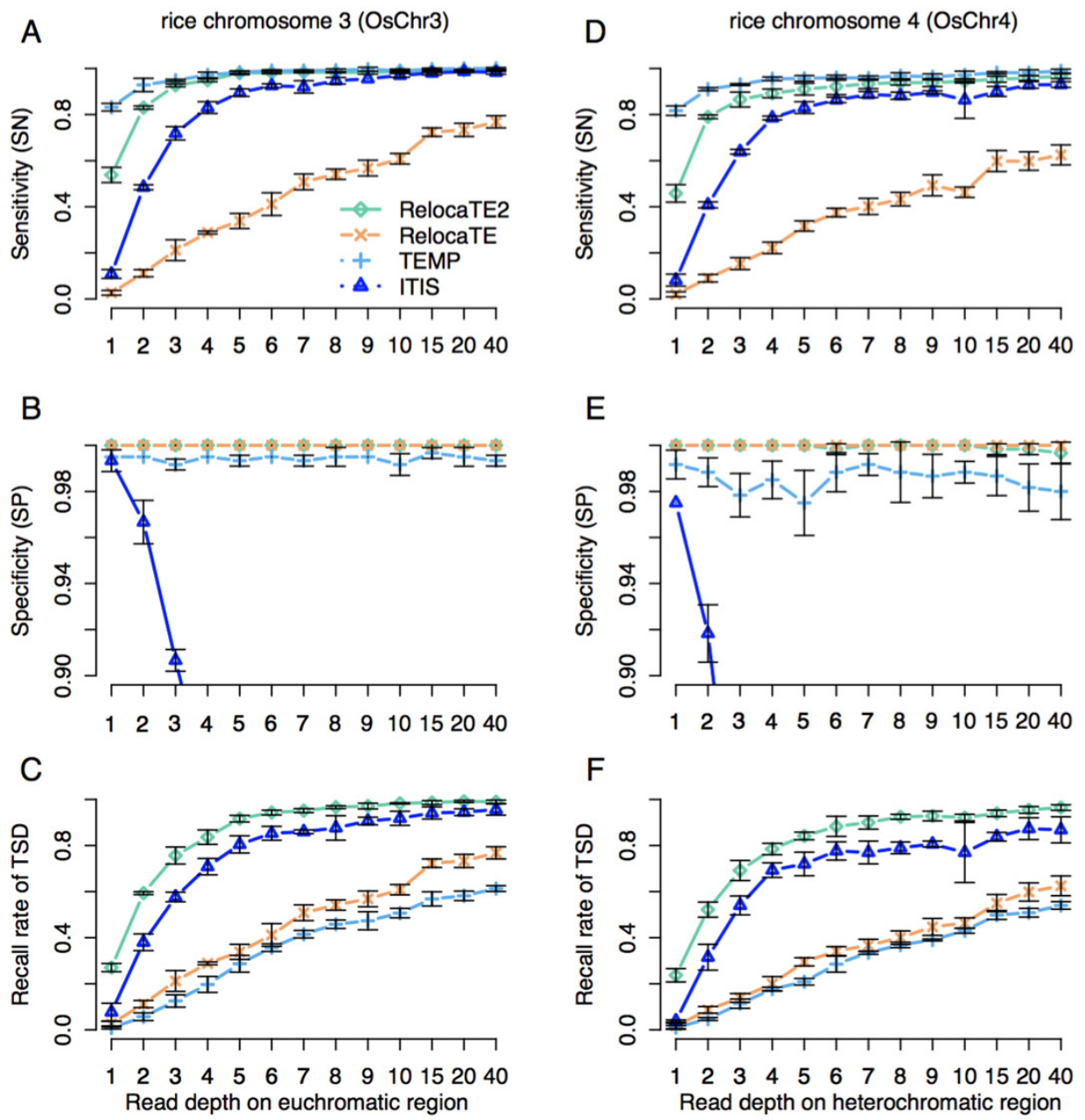

360

361

Figure 2. Performance of RelocaTE2, RelocaTE, TEMP and ITIS on simulated rice data.

362 Comparison of tool performance on rice chromosome 3 for Sensitivity (A) Specificity (B) Recall

363 rate of TSD (C) and comparison of performance on rice chromosome 4 for Sensitivity (D),

364 Specificity $(\mathbf{E})$, Recall rate of TSD $(\mathbf{F})$. Simulations of 200 random transposable element (TE)

365 insertions were generated for rice chromosome 3 (OsChr3) and rice chromosome 4 (OsChr4)

366 with three replicates. A series of datasets with varying sequence depths (from 1 to 40) were

367 generated for each simulation dataset. Sensitivity (SN), Specificity (SP) and Recall rate of target

368 site duplication (TSD) of each tool were estimated for each of these datasets and plotted against

369 sequence depth. The error bars show the standard deviation of three replicates with different sets 370 of 200 random TE insertions. SN was defined as the percentage of calls within 100 base pairs of

371200 random TE insertions. SP was defined as the percentage of calls not within 100 base pairs of

372200 random TE insertions. Recall rate of TSD was defined as the percentage of true positive

373 calls that correctly matched the simulated TSD of TE insertions. 

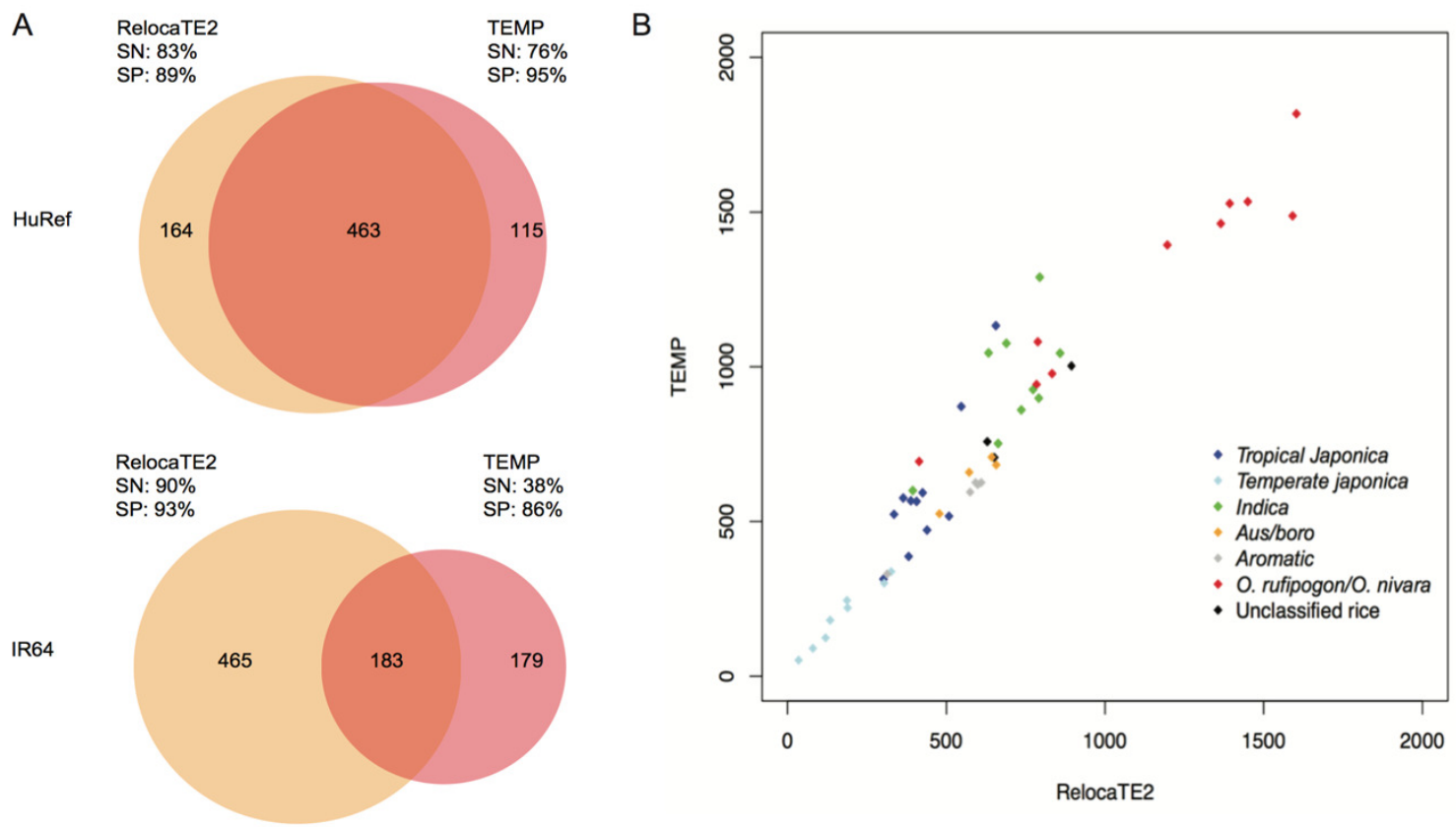

374

375 Figure 3. Performance of RelocaTE2 and TEMP on biological dataset in HuRef genome,

376 IR64 genome, and 50 rice and wild rice strains. A. Venn diagram of the overlap in non-

377 reference TE insertions identified in the HuRef genome and the rice IR64 genome using

378 RelocaTE2 and TEMP. Sensitivity (SN) and Specificity (SP) were assessed by comparing the

379 assembled HuRef genome to the GRCh36 reference genome and the assembled IR64 genome to

380 the MSU7 reference genome. SN was defined as the percentage of validated calls out of all

381 validated calls by either RelocaTE2 or TEMP. SP was defined as the percentage of validated

382 calls out of all calls by each tool. B. Comparison of the number of non-reference TE insertions of

38314 TE families in 50 rice and wild rice strains identified by RelocaTE2 and TEMP. Strains are

384 color-coded based on subpopulation classification. 